\title{
Sudden cardiac arrest risk in young athletes
}

\author{
P J-L Gradidge, ${ }^{1}$ MSc (Med) (Biokinetics); D Constantinou, ${ }^{1} \mathrm{MB}$ BCh, BSc Med (Hons), FFIMS; L Goldberg, ${ }^{2}$ MD, PhD, FESC \\ ${ }^{1}$ Centre for Exercise Science and Sports Medicine, School of Therapeutic Sciences, Faculty of Health Sciences, University of the Witwatersrand, \\ Johannesburg, South Africa \\ ${ }^{2}$ Department of Cardiology, Wits Donald Gordon Medical Centre, Johannesburg, South Africa
}

Corresponding author: P J-L Gradidge (philippe.gradidge@wits.ac.za)

\begin{abstract}
Underlying cardiac abnormalities are the main cause of unexpected death in athletes on field. These abnormalities have been associated with a previous history of syncope, a family history of sudden cardiac arrest (SCA), cardiac murmur, a history of over-exhaustion post exercise and ventricular tachyarrhythmia during physical activity. The timely diagnosis of susceptible athletes may assist with an appropriate management plan for these individuals, and allow for the prevention of premature death in sport. A young football player was screened for SCA risk using the fundamental components of the pre-participation examination (PPE) - essentially, a medical history, a resting and stress electrocardiogram, and an echocardiogram to support clinical findings. The case is submitted with consideration of the applicable literature to accentuate the importance of using PPE to prevent SCA in young athletes.
\end{abstract}

S Afr J SM 2013;25(2):53-54. DOI:10.7196/SAJSM.469

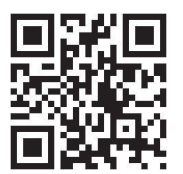

The foremost cause of death on field in children and young athletes is sudden cardiac arrest (SCA), with an increased number of cases worldwide over the past decade. ${ }^{[1]}$ Although SCA prevalence is relatively low in the global adult athletic community (1/65 000 - 1/200 000), it represents a large majority (75\%) of deaths during exercise. ${ }^{[1,2]}$ The Fédération Internationale de Football Association (FIFA) is leading the drive to ensure that the pre-participation examination (PPE) - the template of which is available on the FIFA website - becomes a mandatory component of a football player's career. ${ }^{[1,3]}$ The key element in the PPE is the resting and stress electrocardiogram (ECG), which is arguably the most vital component in identifying SCA risk. ${ }^{[1]}$

Use of the PPE would serve to avoid future SCA incidences among amateur and professional football players alike. The most recent example of SCA was that of the high-profile footballer, Fabrice Muamba, who collapsed suddenly on field at age 23 years; after cardiac arrest that lasted 78 minutes, he was resuscitated and consequently discharged from hospital several weeks later. ${ }^{[4]}$ On the other hand, a medical team failed to revive Italian footballer, Piermario Morosini, following SCA. ${ }^{[5]}$ These incidences of SCA and sudden cardiac death (SCD) could have been avoided; in the former case, by the use of PPE, and in the latter, by having adequately trained staff present on field.

Maron's ${ }^{[6]}$ comprehensive review on the policy regarding the prevention of unexpected mortality in hypertrophic cardiomyopathy adds valuable insight into the use of PPE as a screening tool. The emphasis of pre-competition medical assessment is on risk stratification; although it is strongly advocated by the football community worldwide, it is not always included as an essential procedure in developing African countries. The main objectives of this study were therefore to emphasise the importance of such risk stratification, using a case study focused on an asymptomatic athlete at risk of SCA as an example.

\section{Case}

A 23-year-old South African male football player, involved in amateurlevel football, was referred for examination. He was a non-smoker who had no health complaints, and claimed good effort tolerance. The individual had never experienced blackouts, typical angina or palpitations. There was no known allergy, he did not drink alcohol and he had no family history (FH) of SCD. However, his mother had a history of hypertension.

On examination, his general appearance was normal. He was not pale and did not show signs of clubbed fingers. Neither enlargement of the thyroid gland nor signs of thyrotoxicosis were present. His jugular venous pulse was not raised. Arterial pulses at all sites of examination were present and he had normal characteristics, with no delays. His heart rate (HR) in the supine position was 42 beats per min (bpm) and irregular. On the other hand, his HR in the standing position was $74 \mathrm{bpm}$ and regular. His resting blood pressure was $110 / 68 \mathrm{mmHg}$. His first heart sound (S1) was normal and there was a short ejection systolic murmur (ESM) of $1-2 / 6$. His second heart sound (S2) occurred with physiological split. A short bruit could be heard over both the carotid arteries and abdominal aorta. His chest was clear and showed no signs of any anomaly. An abdominal examination proved unremarkable and no peripheral oedema was present.

\section{Investigations and key findings}

Resting ECG

The individual's sinus rhythm was $42 \mathrm{bpm}$; Mobitz 1 (Wenckebach phenomenon) 3:2 and 2:1 atrioventricular block (AVB) were found in the supine position, as well as early repolarisation syndrome. An 
ECG was done in the prone position, showing an HR of $74 \mathrm{bpm}$, 1st AVB and Mobitz 1 AVB.

\section{Stress ECG}

When undergoing an effort ECG, the participant reached peak exercise levels at the fifth stage of the Bruce protocol when the test was terminated, specifically at 14:37 min of exercise, corresponding to 17.1 metabolic equivalnts. His peak HR was $193 \mathrm{bpm}$ (98\% of predicted maximum) and he exhibited no chest pain, ischaemic ECG changes, conduction abnormalities or early repolarisation during the exercise stress test.

\section{Echocardiogram}

A normal-size aorta was found, with no aortic co-arctation. He had a normal 3-cusp aortic valve. The left atrium was $3.5 \mathrm{~cm}$ and the intraventricular septum of the left ventricle was $0.99 \mathrm{~cm}$ in the mid-segment. The end-diastolic dimension/end-systolic dimension was $4.9 / 2.9 \mathrm{~cm}$ and the ejection fraction was 0.72 . There were no regional wall motion abnormalities. There was mild degeneration of the medial cusp of the anterior mitral valve leaflet associated with minimal mitral regurgitation. The right ventricle, right atrium and pulmonary artery, however, were normal. There was trace pulmonary regurgitation and tricuspid regurgitation. His pulmonary arterial pressure was $21.7 \mathrm{mmHg}$.

\section{Discussion}

Examination revealed clear signs of cardiac anomalies in an otherwise asymptomatic, fit, young athlete. This 23-year-old male football player had features of athletic heart: prominent bradycardia, AVB $1-2$ degree (Mobitz 1) and early repolarisation syndrome. There was a possible degree of atrioventricular conduction abnormalities, which is somewhat unusual; it was suggested that he should concentrate on skills development rather than on endurance training. His ESM was assessed as being functional. However, in light of the aforementioned anomalies, blood tests should be conducted, including thyroid stimulating hormone, full blood count, N-terminal ProB-type natriuretic peptide (NT-ProBNP) and iron studies. The mild mitral valve prolapse would require antibiotic prophylaxis in certain situations, such as dental procedures.

The majority of documented cases of SCA and SCD occurred in asymptomatic athletes such as presented in this case; nevertheless, there was clear evidence of cardiac anomalies and potential risk. Recently published research showed that $61 \%$ of young urban football players were at risk of SCA, even though they showed no physical evidence of predisposition. ${ }^{[7]}$ Interestingly, some of the underlying causes of SCA risk that were stressed in the study included: cardiac risk factors, such as cardiac murmur (8\%); a history of fainting during sporting activity (21\%); and an FH of SCA (11\%). ${ }^{[7]}$ Similarly, a survey of healthy Italian young male athletes found that about $2 \%$ of the sample had underlying cardiac anomalies with the potential for future cardiac conditions. ${ }^{[8]}$ The findings of these and other studies confirm that asymptomatic athletes may have hidden characteristics that could ultimately predispose the athletic population to severe cardiac events during exercise. A comprehensive PPE would enable such athletes to participate in physical activity and exercise with an awareness of the underlying abnormalities, essentially serving to evade future premature mortality on field.

It the case described here, it is relevant to have the detailed evaluation of the player; should he end up with any problem, or be re-assessed, there is a point of reference for comparative purposes and to determine whether further pathology has developed.

\section{Conclusion}

Use of an ECG as an essential component of the medical assessment may result in a lower incidence of SCA and consequent death in athletes. Failure to perform routine PPEs among athletes can have tragic consequences. We recommend that exclusive medical attention be offered to all amateur and elite athletes in all sporting codes, particularly in individuals with signs indicative of risk. The use of an ECG in the screening process alone is not sufficient to preclude SCA, but should form part of a battery of tests. These would ideally prevent potential false-positive evaluation results that may initiate further superfluous assessments and tests. A cross-discipline approach to PPE in sport should be developed using the FIFA approach, ${ }^{[1]}$ but this needs to have a strong evidence base before implementation in nonfootball-related disciplines.

\section{References}

1. Kramer E, Dvorak J, Kloeck W. Review of the management of sudden cardiac arrest on the football field. Br J Sports Med 2010;44(8):540-545. [http://dx.doi.org/10.1136/ bjsm.2010.074526]

2. Harmon KG, Asif IM, Klossner D, Drezner JA. Incidence of sudden cardiac death in national collegiate athletic association athletes. Circulation 2011;123:1594-1600. [http://dx.doi.org/ 10.1161/CIRCULATIONAHA.110.004622]

3. F-MARC. FIFA pre-competition medical assessment (PCMA). http://fifa.com (accessed 3 April 2013).

4. Redhead J, Gordon J. Emergencies in Sports Medicine. Oxford: Oxford University Press, 2012.

5. Sky News. Footballer Dies After Collapsing On Pitch. http://news.sky.com/ story/10158/footballer-dies-after-collapsing-on-pitch (accessed 3 April 2013).

6. Maron BJ. Contemporary insights and strategies for risk stratification and prevention of sudden death in hypertrophic cardiomyopathy. Circulation 2010;121:445-456. [http:// dx.doi.org/ 10.1161/CIRCULATIONAHA.109.878579]

7. Gradidge PJ, Constantinou D, Fiddes J, Hoosain M, Williams M. Sudden cardiac arrest risk profiling of urban Johannesburg footballers. South African Journal for Physical, Health Education, Recreation and Dance 2012;Dec (Suppl 2):247-254.

8. Rizzo M, Spataro A, Cecchetelli C, et al. Structural cardiac disease diagnosed by echocardiography in asymptomatic young male soccer players: Implications for preparticipation screening. Br J Sports Med 2012;46:371-373. [http://dx.doi.org/10.1136/ bjsm.2011.08569] 